\title{
How proteins are transported into mitochondria
}

\author{
Walter Neupert and Gottfried Schatz
}

\begin{abstract}
Most mitochondrial polypeptides are synthesized outside the organelle as precursors which are usually larger than the 'mature' polypeptides found within mitochondria. The precursors are imported into the mitochondria by a process which is independent of protein synthesis but dependent on high-energy phosphate bonds inside the mitochondria. This mechanism is basically different from that which governs the movement of secretory polypeptides across the membrane of the endoplasmic reticulum.
\end{abstract}

How are mitochondria made? They contain their own genetic system that makes the mitochondrial RNAs and a few mitochondrial polypeptides such as the three largest subunits of cytochrome oxidase, the cytochrome $b$ apoprotein, two subunits of the ATPase complex and one protein of the $30 \mathrm{~s}$ mitochondrial ribosomal subunit ${ }^{1}$. However, these polypeptides account for less than $10 \%$ of the total protein mass of a mitochondrion. All the other several hundred mitochondrial polypeptides are coded by nuclear genes, translated on cytoplasmic ribosomes and imported into the mitochondrion. This import process must be highly specific since each mitochondrial polypeptide occupies a precisely defined location within the organelle. Mitochondria (Fig. 1) consist of two distinct membranes: an outer membrane (whose function is still somewhat obscure) and a highly infolded inner membrane (which houses the machinery for oxidative phosphorylation). The space between the outer and inner membrane is the 'intermembrane space'; the space bounded by the inner membrane is the 'matrix space'.

The massive movement of polypeptides into the mitochondria raises several intriguing questions. Why is a newly made mitochondrial protein only imported into mitochondria and not into other organelles? How is an imported polypeptide steered to its proper intramitochondrial location? Why do the enzymes destined to be imported not become active in the cytosol? How are water-insoluble membrane proteins transported? How is the transport regulated?

Walter Neupert is at the Institute of Physiological Chemistry, University of Götingen, German Federal Republic; Gottfried Schatz is at the Biocenter, University of Basel, Switzerland.

\section{Protein traffic in the eukaryotic cell}

In addition to transporting proteins into mitochondria. a typical eukaryotic cell must also transport proteins across other intracellular membranes and proteins must be inserted into membranes such as the endoplasmic reticulum and the plasma membrane. One of the simplest hypotheses to account for ordered 'protein traffic' is that each protein carries in itself a specific structure that recognizes a complementary structure on the membrane across or into which it has to move. A similar principle might govern the assembly of oligomeric proteins.

\section{Secretion of proteins is coupled to protein synthesis}

The secretion of proteins by cells is closely related to intracellular protein traf$\mathrm{fic}^{2}$. In eukaryotes, the synthesis of a protein destined to be secreted occurs on a ribosome which is attached to the cytoplasmic side of the endoplasmic reticulum: protein synthesis and translocation are

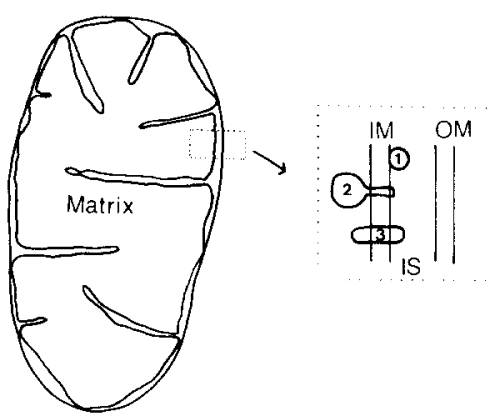

Fig. 1 . The structure of a mitochondrion. $I M=$ inner membrane; $O M=$ outer membrane; IS = intermem brane space; 1 = cytochrome c; 2 = oligomvcin sensitive ATPase complex; $3=A D P / A T P$ carrier. obligatorily linked. To explain the specificity of transport, it has been suggested that the amino terminal region of the nascent polypeptide recognizes some hypothetical receptor on the target membrane ${ }^{3.4}$.

This process has two salient features: Firstly, transmembrane movement is 'cotranslational'; it is coupled to elongation of the polypeptide chain. As a result, this translocation process is often termed 'vectorial translation'. Secondly, the protein is immediately sequestered on the opposite side of the membrane; the completed polypeptide chain is thus probably never present in the compartment where it is synthesized.

\section{Mitochondrial protein import is not obligately coupled to protein synthesis}

Do mitochondria import proteins by vectorial translation? Apparently not, since neither of the two criteria for vectorial translation is met. This was first indicated by studies in vivo ${ }^{5,6}$ which established: (1) pulse-labelled mitochondrial proteins are initially found outside the mitochondria; (2) newly made mitochondrial proteins can be chased into mitochondria only after a lag; (3) this lag differs for different pulse-labelled proteins; (4) inhibition of protein synthesis by cycloheximide fails to prevent the appearance of pulse-labelled proteins inside the mitochondria.

Clearly, these observations do not support a typical cotranslational mechanism Instead, they open the possibility that import is independent of concomitant translation and proceeds via extramitochondrial pools of mitochondrial proteins. Further evidence for a post-translational mechanism came from subsequent studies with cell-free systems. On the one hand, 
these experiments demonstrated clearly that the synthesis of a mitochondrial protein can be separated from its transport into the mitochondria in time as well as space. On the other hand, the cell-free systems showed that all imported mitochondrial proteins studied so far are initially made as precursors which are qualitatively different from their functional counterparts inside the mitochondria.

\section{Precursors of mitochondrial proteins made in the cytoplasm}

If cytoplasmically-made mitochondrial proteins are synthesized in heterologous cell-free systems and isolated by immunoprecipitation. most but not all of them display an apparent molecular weight which is several thousand daltons larger than that of the corresponding mature proteins*. The precursors which are larger than the mature polypeptides probably have additional sequences at the amino terminus. So far, several dozen mitochondrial precursors have been identified; a few representative examples are listed in Table $I$.

It has been reported that cytoplasmically-made subunits of oligomeric proteins such as cytochrome oxidase are made as a polyprotein which is imported and cut to the mature subunits inside the mitochondria ${ }^{16}$. However, recent studies failed to confirm this; in fact, the individual cytoplasmically-made subunits of cytochrome oxidase and ATPase are made as discrete precursors which are only a few thousand daltons larger than the mature subunits ${ }^{17.18,19}$. In several cases the larger molecular weight precursors detected in the cell-free translation systems can also be found in pulse-labelled intact cells (Table I). The kinetic behaviour of these larger forms upon a chase was consistent with their role as precursors to the corresponding 'mature' polypeptides.

\section{Import in vitro}

Yeast or Neurospora mitochondria can incorporate mitochondrial precursors, synthesized in a cell free system, in the absence of protein synthesis ${ }^{7.12-15}$. This appears to be a specific process: First, it is accompanied by proteolytic removal of any extra sequences which had been present in the precursor. Second, the resulting mature polypeptide (but not any residual precursor) becomes resistant to externally added proteases. Third. non-mitochondrial proteins synthesized in vitro or radiolabelled mature mitochondrial proteins are

* It is impossible to cite all reports on mitochondrial precursor polypeptides in this brief review. A few typical examples are listed in Table $\boldsymbol{I}$.

TABI.E I.

A few representative precursors to cytoplasmically-made mitochondrial proteins.

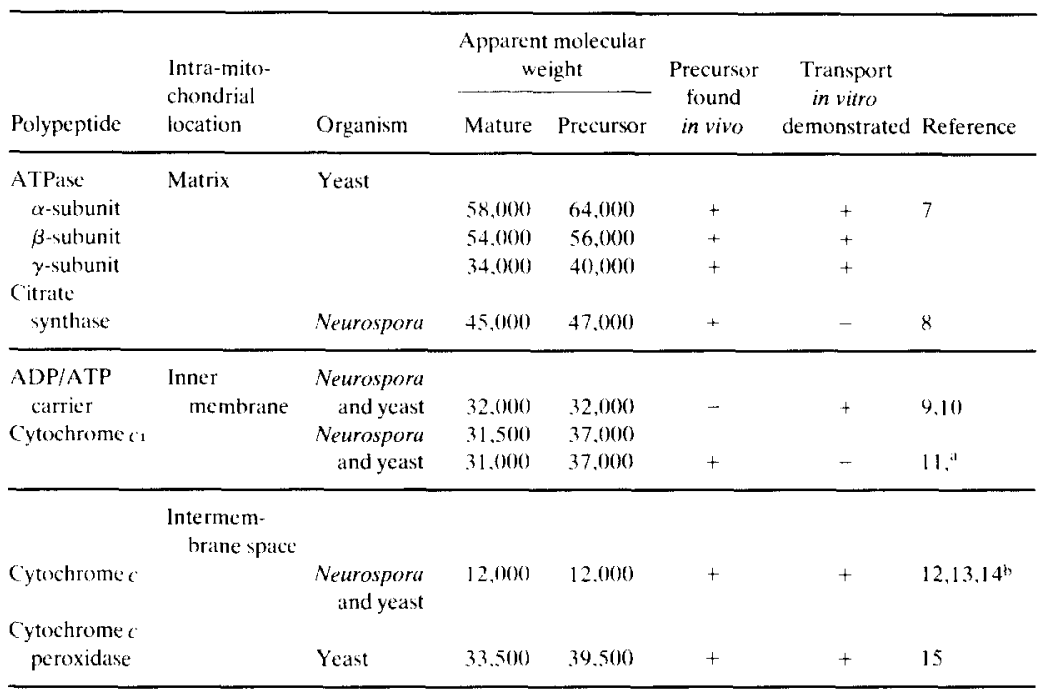

${ }^{a}$ R. Zimmermann, H. Weiss and W. Neupert, unpublished work.

h. Gasser and G. Schatz. unpublished work.

neither altered nor rendered proteaseresistant by isolated mitochondria. Figs. 2 and 3 illustrate import in vitro of precursors with or without amino te rminal extensions.

\section{Mitochondrial precursors are synthesized on free polysomes}

The available evidence strongly suggests that precursors of mitochondrial proteins are predominantly synthesized on free polysomes (see Refs 9, 12, 20, also M. Suissa and G. Schatz, unpublished work). In one type of experiment, the different classes of cytoplasmic polysomes were first isolated from different cell types and allowed to complete their nascent polypeptide products in vitro ('read-out); in the second type of experiment, the polysome-associated mRNA was isolated and translated in a reticulocyte lysate. In both instances, the radiolabelled translation products were checked for mitochondrial precursors by immunoprecipitation. Most of the mRNA for mitochondrial proteins was found to be associated with free polysomes. regardless of whether the mitochondrial protein tested for was a membrane protein or a matrix protein.

However, the situation may be more complex, at least in yeast. If yeast mitochondria are isolated under conditions preventing degradation of polysomes, they contain cytoplasmic polysomes bound to their surface ${ }^{21}$. Compared to free polysomes, these mitochondria-associated polysomes are enriched in mRNA for mitochondrial proteins (M. Suissa and G.
Schatz, unpublished work). Nevertheless, more than $90 \%$ of the total mRNA for yeast mitochondrial proteins is associated with free polysomes, indicating that these polysomes make the bulk of the imported mitochondrial proteins. This result, together with the observations mentioned earlier, makes it extremely unlikely that the mitochondria-associated polysomes found in yeast represent the obligate port of entry for polypeptides into mitochondria. The biological significance of these mitochondria-bound polysomes remains unknown.

\section{In what form do precursor proteins occur outside mitochondria?}

Mitochondrial proteins synthesized in heterologous translation systems are always released into the post-ribosomal supernatant fraction. This is not only true for soluble matrix proteins but also for membrane proteins such as the ADP/ATP carrier or subunit $\mathrm{V}$ of cytochrome oxidase. The precursors discharged into the supernatant are accessible to added protease or antibody. Thus, precursors synthesized in vitro are apparently neither enclosed in membrane vesicles nor deeply buried in a lipid phase. Similarly, after pulse-labelling intact yeast cells, at least $50 \%$ of the precursor to cytochrome oxidase subunit $V$ is in the 'cytosolic' fraction (A. Lewin and G. Schatz, unpublished work). These observations suggest that mitochondrial protein precursors are initially released into the cytosol. However, it is difficult to exclude 
that the precursors are first discharged into some highly fragile vesicle which are disrupted when the cells are homogenized.

How can precursors to insoluble membrane proteins exist in the cytosol? We do not know, but a key to this question may be provided by observations from our laboratories that the precursors to the ADP/ATP carrier or to cytochrome oxidase subunit $V$ do not exist as monomers but as larger aggregates. It is not known whether these aggregates are simply homo-oligomers of the precursor or complexes between precursor and other proteins. Preliminary observations suggest, moreover, that precursors to some mitochondrial membrane proteins are amphipathic. This raises the possibility that the precursor aggregates are protein micelles in which the hydrophobic domains are buried in the interior.

\section{The molecular mechanism of import}

What are the molecular events that transport a polypeptide into mitochondria?

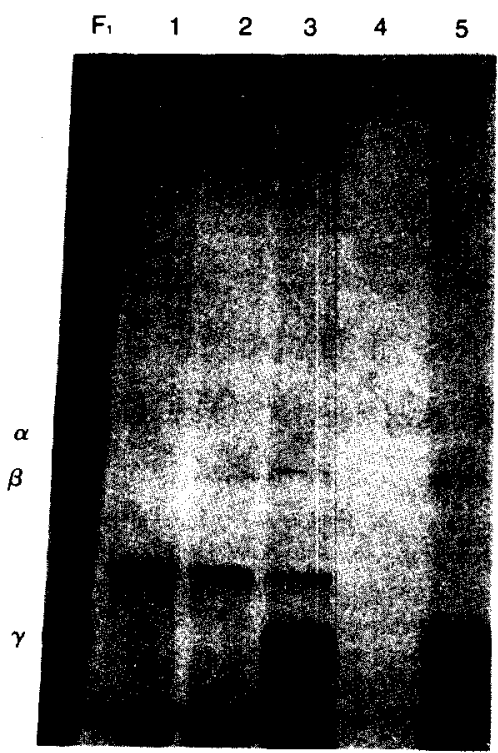

Fig. 2. The precursor to the y-subunit of yeas mitochondrial $F_{1}$ ATPase is processed and imported into mitochondria in vitro. A radioautogram of an $S D S$-polyacrylamide gel is shown. The track labelled $F_{1}$ contains radiolabelled mature $F_{1}-A$ TPase from yeast as a standard. Track 1: $\gamma$-subunit precursor synthesized in vitro. Track 2: after labelling in vitro, the reticulocyte lysate was inhibited with cycloheximide and incubated for 60 min at $29^{\circ} \mathrm{C}$ with isolated yeast mitochondria: the mitochondria were then spun down and the supernatamt was subjected to immunoprecipilation with anti- $\gamma$ serum. Track 3: same as track 2 except that the mitochondrial pellet was analysed. Track 4: same as track 2 except that the lysate-mitochondria mixture was treated with trypsin and chymotrypsin before removal of the mitochondria; the mitochondria-free supernatant was analysed. Track 5: same as track 4 except that the mitochondrial pellet was analysed.

\section{supernatant mitochondria}

\section{mitochondria}

$(+$ proteinase)

$0^{\prime} \quad 1^{\circ} 30^{\circ} \quad 60^{\circ} \quad 0^{\circ} \quad 15^{\circ} 30^{\circ} 60^{\circ} \quad 0^{\circ} \quad 15^{\circ} 30^{\circ} \quad 60^{\circ}$

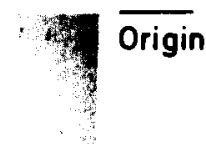

Fig. 3. The precursor to the ADP/ATP carrier is imported into Neurospora mitochondria without proteolytic processing. A reticulocyte lysate containing radiolabelled precursor to the ADP/ATP carrier from Neurospora was incubated with isolated Neurospora mitochondria. Aliquots were withdrawn at the times indicated and freed from mitochondria by centrifugation. The supernatant was saved for analysis (cf. below). The mitochondrial pellets were resuspended and one half of each suspension was treated with proteinase $K$ to degrade accessible proteins, followed by inactivation of the proteinase by phenyl methyl sulfonyl flworide. Finally, all mitochondrial pellets were solubilized with Triton X-100 and the Triton extracts as well as the mitochondria-free supernatants (cf. above) were subjected to immunoprecipitation with an antiserum against the ADP/ATP carrier. The immunoprecipitates were resolved on SDS-polyacrylamide gels and radioautographed.

Is there a single mechanism for all proteins or are there different mechanisms for proteins transported into different locations within the organelle? We cannot yet answer these questions, but we can present some ideas as to how import might work. Step 1: The polypeptide destined for import into mitochondria is made on free polysomes as a precursor which differs from the mature polypeptide in conformation and (in most cases) in molecular weight. The precursor is then discharged into a cytosolic pool whose size may vary depending on the metabolic conditions. The evidence for this is quite good and has already been discussed

Step 2: The precursor binds to a 'receptor' on the mitochondrial surface. Most likely, each precursor polypeptide contains a domain which recognizes some structure on the cytoplasmic surface of the mitochondrial outer membrane. There is indeed some evidence that mitochondrial precursors can specifically bind to isolated mitochondria, that this binding is temperature- and energy-independent ${ }^{22}$ and that it is abolished if the mitochondria are stripped of their outer membranes (D. L. Sabatini, personal communication). At present, there are no data proving the existence of distinct 'import receptors' on the mitochondrial surface, but we suspect that there exist at least two classes of such receptors: one class for proteins transported only across the outer membrane and the second class for proteins trans- ported across both membranes. This second class of receptors might be located at those sites on the outer membrane where the inner and outer membrane are closely apposed (Fig. 1).

Step 3: Interaction between the precursor and its corresponding receptor opens up a pore which allows at least partial diffusion of the precursor across one or both membranes. This step requires high-energy phosphate bonds. The evidence for this is as follows: if ATP in the mitochondrial matrix of intact yeast cells is depleted by suitable mutations or inhibitors, import of proteins into mitochondria stops and unprocessed precursors accumulate in the cytosol $^{11}$. The energy-dependent step is import, not proteolytic processing: on the one hand. energy-dependence of import was demonstrated for the precursor of ADP/ATP carrier ${ }^{22}$ which has the same molecular weight as the mature protein; on the other hand, the processing protease (cf. below) does not require ATP. The energy-dependence of import by isolated Neurospora mitochondria has also been demonstrated in vitro: carbonyl cyanide $m$-chlorophenylhydrazone (CCCP), which apparently lowers the intramitochondrial ATP concentration, blocks import of mitochondrial protein precursors.

Step 4: The precursor is converted to the mature polypeptide on the opposite side of the membrane barrier. The resulting conformational change could then trap the polypeptide inside the mitochondria and 
thereby provide a driving force for unidirectional import. Almost certainly, step 4 differs for different precursors. This must be anticipated because not all precursors are proteolytically processed to the mature polypeptide. Differences might exist even among those precursors that are not proteolytically processed. For example: with cytochrome $c$, the heme-free precursor polypeptide is probably fitted with its heme group in the intermembrane space and thereby converted to the mature holocytochrome $c$ which then attaches to the outer face of the mitochondrial inner membrane ${ }^{12.13 .14}$. The precursor to the ADP/ATP carrier (which differs from the mature polypeptide with respect to solubility and chromatographic properties, but not molecular weight - see Table I and Fig. 3) might be converted to its mature conformation on being inserted into the hydrophobic core of the mitochondrial inner membrane and subsequent dimerization. Finally, precursors that carry aminoterminal extensions are proteolytically processed by an intramitochondrial protease. Removal of the extra amino acid sequences might trigger an essentially irreversible conformational change which exposes sites necessary for catalytic function or assembly with partner polypeptides ('vectorial processing").

A mitochondrial protease that specifically processes yeast mitochondrial precursors has recently been identified and purified several hundredfold from yeast ${ }^{23}$. The protease is water-soluble, sensitive to $o$-phenanthroline and EDTA but insensitive to most inhibitors of serine proteases. It cleaves mitochondrial precursors to their mature size (but no further) and is inactive towards all non-mitochondrial proteins tested. Since it is localized in the matrix space, its role in vivo is probably directed at those precursors that are transported across both mitochondrial membranes. This implies that the intermembrane space, too, should contain its own distinct protease for processing proteins (such as cytochrome $c$ peroxidase) which are transported into this space.

\section{What next?}

A beginning has been made but we do not yet really understand how the precursor polypeptides are translocated across the mitochondrial membrane(s). To know more we must isolate and study the molecules that participate in this translocation process. In addition to the processing proteases we have to isolate chemically significant amounts of precursors and the (still hypothetic) mitochondrial receptor molecules. In the long run, it should be possible to study the internalization of mitochondrial precursors with liposomes which contain a processing protease in their lumen and a mitochondrial import receptor' inserted into their phospholipid bilayer. Even if this could be achieved. there would still be some lingering doubt as to whether such experiments in vitro faithfully reflected the situation within a living cell. Such doubts could perhaps be silenced by the discovery of temperature-sensitive mutants in which specific steps of the import process are blocked. Since genetic studies are most conveniently performed with microbial systems, we suspect that Neurospora and yeast will continue to feature prominently in future efforts to understand how mitochondria import proteins from the cytoplasm.

\section{References}

1 Schatz, G. and Mason, T. L. (1974) Ann. Rev. Biochem. 43, 51-87

2 Palade. G. W. (1975) Science 189, 347-358

3 Milstein, C.. Brownlee, G. G., Harrison. T. M. and Matthews, M. B. (1972) Nature New Biology 239. 117-120

4 Blobel, G. and Dobberstein. B. (1975) J. Cell Biol. 67, 835-851

5 Hallermayer, G., Zimmermann, R. and Neupert. W. (1977) Eur.J. Biochem. 81, 523-532

6 Schatz, G. (1979) FEBS Letters 103, 201-211

7 Macecenini. M.-L., Rudin, Y.. Blobel, G. and Schatz. G. (1979) Proc. Natl. Acad. Sci. U.S.A. $76.343-347$
8 Harmey, M. A. and Neupert, W. (1979) FEBS Letters $108,385-389$

9 Zimmermann, R., Paluch, U., Sprinzl, M. and Neupert. W. (1979) Eur. J. Biochem. 99, 247-252

10 Nelson, N. and Schatz. G. (1979) in: Membrane Bioenergetics (Lcc. C. P.. Schat7, G. and Ernster, 1...eds), pp. 133-152. Addison-Wesley, Reading

11 Nelson. N. and Schatz. G. (1979) Proc. Natl. Acad. Sci. U.S.A. 76. 4365-4369

12 Zimmermann, R.. Paluch, U. and Neupert. W. (1979) FEBS Letters 108, 141-151

13 Korb, H. and Neupert. W. (1978) Eur. J. Biochem. 91. 609-620

14 Neher, F.-M., Harmey, M. A.. Hennig, B., Zimmermann, R. and Neupert. W. (1980) in Structure and expression of the mitochondrial genome (Kroon, A. M. and Saccone. C.. eds) , 413-422. North-Holland. Amsterdam

15 Maccechini. M.-I.., Rudin, Y. and Schatz, G. (1979) J. Biol. Chem. 254, 7468-7471

16 Poyton, R. O. and McKemmic, E. (1979) J. Biol. Chem. 254,6763-6771

17 Lewin, A., Gregor, I., Mason, T. L., Nelson, N and Schatz, G. (1980) Proc. Natl. Acad. Sci. U.S.A. $77,3998-4002$

18 Mihara. K. and Blobel, G. (1980) Proc. Natl. Acad. Sci. U.S.A. 77, 4160-4164

19 Schmelzer, E. and Heinrich, P. C. (1980) J. Biol. Chem. 255. 7503-7506

20 Raymond. Y. and Shore, G. S. (1979) J. Biol Chem. 254, 9335-9338

21 Kellems. R. E., Allison, V. F. and Butow, R. A (1975) J. Cell Biol. 65, 1-14

22 Zimmermann, R. and Neupert. W. (1980) Eur.J. Biochem. 109.217-229

23 Böhni, P., Gasser, S., Leaver, C, and Schatz, (; (1980) in Structure and expression of the mitochondrial genome (Kroon, A. M. and Saccone, $C$., eds). pp. 42.3-4.33, North-Holland. Amsterdam

\title{
Protein crystals as a model for mitochondrial matrix proteins
}

\author{
Paul A. Srere
}

Information on the structure of protein crystals and the activity of crystalline enzymes may be used to help understand the structure of the mitochondrial matrix and the activity of the proteins therein.

I have recently reviewed in $T I B S^{1}$ the evidence that indicates that the rat liver mitochondrial matrix contains a high concentration (approximately $56 \% \mathrm{w} / \mathrm{w}$ ) of closely packed protein molecules ${ }^{2.3}$. I showed that a model consisting of spherical protein molecules packed in a cubical array (six protein-protein contact points per molecule) would represent a protein concentration of $59.76 \%(\mathrm{w} / \mathrm{w})(0.674 \mathrm{~g}$ of $\mathrm{H}_{2} \mathrm{O} / \mathrm{g}$ protein). The protein in this model would occupy $52.36 \%$ of the volume of the

Paul A. Srere is at the Pre-Clinical Science Unit. Veterans. Administration Medical Center, Dallas, Texas 75216 and the Department of Biochemistry. The University of Texas Health Science Center at Dallas, 5323 Harry Hines Boulevard, Dallas, Texas 75235. U.S.A. array. I now wish to extend these ideas to point out the similarity of such an array to the situation that occurs in protein crystals. The information available for the structure of protein crystals and the activity of crystalline enzymes may then be used to help understand the structure of the mitochondrial matrix and the activity of the proteins therein.

Protein crystals are known to contain about $50 \%$ water $(40-60 \%)^{4.5}$ and, for some proteins studied, contact points with other protein molecules in the crystal have been reported as 5 to $10^{6}$. Since these figures agree with those of our model outlined above, protein crystals and their properties may represent an acceptable paradigm for the proteins of the mitochondrial matrix. 